\title{
BMJ Open Anthropometric measurements of general and central obesity and the prediction of cardiovascular disease risk in women: a cross-sectional study
}

\author{
Louise G H Goh, ${ }^{1}$ Satvinder S Dhaliwal, ${ }^{1}$ Timothy A Welborn, ${ }^{2}$ Andy H Lee, ${ }^{1}$ \\ Phillip R Della ${ }^{3}$
}

To cite: Goh LGH, Dhaliwal SS, Welborn TA, et al. Anthropometric measurements of general and central obesity and the prediction of cardiovascular disease risk in women: a cross-sectional study. BMJ Open 2014;4:e004138. doi:10.1136/bmjopen-2013004138

- Prepublication history for this paper is available online. To view these files please visit the journal online (http://dx.doi.org/10.1136/ bmjopen-2013-004138).

Received 27 September 2013 Revised 23 December 2013 Accepted 14 January 2014

CrossMark

\begin{abstract}
${ }^{1}$ School of Public Health, Curtin Health Innovation Research Institute, Curtin University, Perth, Western Australia, Australia

${ }^{2}$ Sir Charles Gairdner Hospital, Hospital Avenue, Nedlands, Perth, Western Australia, Australia ${ }^{3}$ School of Nursing and Midwifery, Curtin Health Innovation Research Institute, Curtin University, Perth, Western Australia, Australia
\end{abstract}

Correspondence to Professor Satvinder S Dhaliwal:

s.dhaliwal@curtin.edu.au

\section{ABSTRACT}

Objectives: It is important to ascertain which anthropometric measurements of obesity, general or central, are better predictors of cardiovascular disease (CVD) risk in women. 10-year CVD risk was calculated from the Framingham risk score model, SCORE risk chart for high-risk regions, general CVD and simplified general CVD risk score models. Increase in CVD risk associated with $1 \mathrm{SD}$ increment in each anthropometric measurement above the mean was calculated, and the diagnostic utility of obesity measures in identifying participants with increased likelihood of being above the treatment threshold was assessed.

Design: Cross-sectional data from the National Heart Foundation Risk Factor Prevalence Study.

Setting: Population-based survey in Australia.

Participants: 4487 women aged 20-69 years without heart disease, diabetes or stroke.

Outcome measures: Anthropometric obesity measures that demonstrated the greatest increase in CVD risk as a result of incremental change, $1 \mathrm{SD}$ above the mean, and obesity measures that had the greatest diagnostic utility in identifying participants above the respective treatment thresholds of various risk score models.

Results: Waist circumference (WC), waist-to-hip ratio (WHR) and waist-to-stature ratio had larger effects on increased CVD risk compared with body mass index (BMI). These central obesity measures also had higher sensitivity and specificity in identifying women above and below the $20 \%$ treatment threshold than BMI. Central obesity measures also recorded better correlations with CVD risk compared with general obesity measures. WC and WHR were found to be significant and independent predictors of CVD risk, as indicated by the high area under the receiver operating characteristic curves $(>0.76)$, after controlling for BMI in the simplified general CVD risk score model.

Conclusions: Central obesity measures are better predictors of CVD risk compared with general obesity measures in women. It is equally important to maintain a healthy weight and to prevent central obesity concurrently.

\section{Strengths and limitations of this study}

- This study provided evidence that anthropometric measures of central obesity are better predictors of cardiovascular disease (CVD) risk compared with general obesity measures in women.

- Central obesity measures add prognostic information on CVD risk in women above the measures of general obesity and should be considered for incorporation into the clinical assessment of CVD risk.

- Although this study is cross-sectional, it is a representative sample of the Australian female population.

- Only one set of baseline measurements is recorded for some risk variables but some important variables are measured twice.

- The predicted 10-year CVD risks are calculated using risk score models to stratify individuals against the treatment thresholds for various risk score models. Prospective data of CVD events were not used.

\section{INTRODUCTION}

In 2008, more than 200 million men and approximately 300 million women were obese. ${ }^{1}$ Overweight and obesity is one of the leading risk factors for mortality, estimated to account for $23 \%$ of the ischaemic heart disease burden. ${ }^{1}$ It results in the deterioration of the entire cardiovascular risk profile. $^{2{ }^{3}}$ Large prospective studies such as the Framingham Heart Study, ${ }^{4}$ the Nurses' Health Study ${ }^{5}{ }^{6}$ and the Buffalo Health Study $^{7}$ have all shown that overweight and obesity are associated with increased cardiovascular disease (CVD) risk. Excess adipose tissue contributes to the cardiovascular and other risks associated with being overweight or obese. ${ }^{8}$ 
The American Heart Association released a Scientific Statement emphasising the importance of assessing adiposity. ${ }^{8}$ New guidelines have also been released by the American College of Cardiology, American Heart Association Task Force on Practice Guidelines and The Obesity Society for the management of overweight and obesity in adults to prevent CVD. ${ }^{9}$ General and central obesity are associated with CVD risk. ${ }^{5}{ }^{10-15}$ Currently used general and central obesity anthropometric measures for assessing adiposity-related risk include: body mass index (BMI; weight in kilograms divided by square of height in meters), waist circumference (WC), hip circumference (HC), waist-to-hip ratio (WHR; ratio of WC to $\mathrm{HC}$ ), waist-to-stature ratio (WSR; ratio of WC to height) and body adiposity index ${ }^{16}$ (BAI; HC divided by height $^{1.5}$, and subtracting 18 from the result). BMI or WC is most commonly used to measure body fatness. ${ }^{10}$

It is, however, unclear which anthropometric measurements are better correlated with CVD risk factors and CVD risk in women, considering adiposity is highly heterogeneous with age, sex and ethnic differences in body fat distribution. ${ }^{8}$ Previous studies have reported that BMI identified individuals at increased risk of CVD as effectively as WC. ${ }^{11}{ }^{12}$ It has also been suggested that BMI is a better predictor of CVD than WC. ${ }^{13}$ Conversely, some studies reported that WC is a better indicator of CVD risk than BMI and WHR, in ethnically diverse groups. ${ }^{14}{ }^{15}$ WC and WHR have also been identified as independent predictors of CVD risk but not BMI, accounting for conventional risk factors in the Framingham risk score model. ${ }^{17}$ More research is thus needed to ascertain which measures are better correlated with CVD risk factors and subsequent CVD risk in women.

We aim to assess the associations between general and central obesity anthropometric measures with CVD risk factors, using a representative sample of 4487 women aged 20-69 years without heart disease, diabetes or stroke. The associations between these indices of obesity with predicted risk calculated from the Framingham risk score model for 10-year CVD incidence or death, ${ }^{18}$ SCORE risk chart for high-risk regions for 10-year CVD death, ${ }^{19}$ general CVD and simplified general CVD risk score models for 10-year CVD incidence and death ${ }^{20}$ were examined. To aid comparison between obesity indices, which are measured in different units, the incremental shift in CVD risk with 1 SD increment in each anthropometric measurement above the mean would be assessed. Finally, we determined which indices of obesity are most sensitive and specific for identifying women at increased 10-year CVD risk.

\section{METHODS}

\section{Study cohort and measurements}

We selected 4487 women aged 20-69 years with no history of heart disease, diabetes or stroke from the population representative sample of 4727 women from the National Heart Foundation (NHF) Risk Factor Prevalence Study. ${ }^{21}$ Participants taking medications to lower their CVD risk factors were also excluded. The participants of the NHF study consisted of residents on the federal electoral rolls of December 1988 in North and South Sydney, Melbourne, Brisbane, Adelaide, Perth, Hobart, Darwin and Canberra in a systematic probability sampling by sex and 5-year age groups. Information on demographic characteristics was collected using a self-administered questionnaire, and conventional CVD risk variables recorded in this prevalence study include anthropometric measures, smoking status, systolic and diastolic blood pressure and lipid levels. Physical measurements of height (to the nearest centimetre), weight (to the nearest 10th of a kilogram) and waist and $\mathrm{HC}$ were collected according to standardised methodologies $^{22} 23$ using two observers. The WC was measured from the front at the narrowest point between the rib cage and iliac crest after full expiration while the $\mathrm{HC}$ was measured from the side at the maximal extension of buttocks by one observer using a metal tape. A second observer recorded another set of measurements and ensured that the metal tape was kept strictly horizontal at all times. The mean of two measurements was taken at each site to the nearest centimetre. Participants were classified as non-smokers, previous smokers or current smokers. ${ }^{21}$ Mercury sphygmomanometers were used to record blood pressure levels on the right arm of seated participants $5 \mathrm{~min}$ apart. ${ }^{21}$ Two readings were taken and the average was used in the analysis. Fasting blood samples were also collected in EDTA tubes and dispatched to the central laboratory at the Division of Clinical Chemistry, Institute of Medical and Veterinary Science, Adelaide each week for lipid levels to be assayed. $^{21}$

\section{Risk score models}

The Framingham 10-year predicted risk for CVD incidence or death was developed using data from the American Framingham Heart Study. ${ }^{18}$ Participants aged 30-74 years who were free of CVD and cancer were included in the model development. The 10-year risk for CVD incidence or death was calculated using these variables: age, sex, systolic blood pressure (SBP), diastolic blood pressure, total cholesterol level, high-density lipoprotein (HDL) cholesterol level, smoking status and diabetes status. The SCORE risk chart was developed by pooling 12 cohort studies to predict the 10-year CVD death risk in Europe. The cohorts consisted of participants aged 19-80 years with no previous history of heart attack. ${ }^{19}$ The SCORE model was derived from a much larger dataset than the Framingham, general CVD and simplified general CVD risk score models. Fewer variables were used in the calculation of the 10-year predicted CVD death risk with the SCORE risk chart for high-risk regions (Denmark, Finland and Norway), ${ }^{19} 24$ these included: age, sex, smoking status, mean total cholesterol level, mean HDL cholesterol level and mean 
Table 1 Characteristics of a representative Australian sample of 4487 women (aged 20-69 years) free of heart disease, diabetes and stroke

\begin{tabular}{|c|c|}
\hline Variables & $\begin{array}{l}\text { Summary } \\
\text { statistics }\end{array}$ \\
\hline \multicolumn{2}{|l|}{ Age (years), n (\%) } \\
\hline $20-29$ & $840(18.7)$ \\
\hline 30-39 & $1116(24.9)$ \\
\hline $40-49$ & $1139(25.4)$ \\
\hline $50-59$ & $743(16.6)$ \\
\hline$\geq 60$ & 649 (14.4) \\
\hline \multicolumn{2}{|l|}{ Ethnicity } \\
\hline Australia & $3329(76.5 \%)$ \\
\hline UK and Ireland & $416(9.5 \%)$ \\
\hline Northern Europe & $180(4.1 \%)$ \\
\hline Southern Europe & $234(5.4 \%)$ \\
\hline Asia & $195(4.5 \%)$ \\
\hline \multicolumn{2}{|l|}{ Smoking status, $\mathrm{n}(\%)$} \\
\hline Non-smoker & $2652(59.1)$ \\
\hline Previous smoker & $880(19.6)$ \\
\hline Current smoker & $955(21.3)$ \\
\hline $\mathrm{SBP}(\mathrm{mm} \mathrm{Hg})$ & $122.1 \pm 18.4$ \\
\hline $\mathrm{DBP}(\mathrm{mm} \mathrm{Hg})$ & $75.7 \pm 10.8$ \\
\hline Total cholesterol (mmol/L) & $5.5 \pm 1.2$ \\
\hline HDL cholesterol (mmol/L) & $1.5 \pm 0.4$ \\
\hline $\begin{array}{l}\text { Ratio of total cholesterol to HDL } \\
\text { cholesterol }\end{array}$ & $3.9 \pm 1.3$ \\
\hline BMI $\left(\mathrm{kg} / \mathrm{m}^{2}\right)$ & $24.8 \pm 4.7$ \\
\hline WC $(\mathrm{cm})$ & $76.2 \pm 11.1$ \\
\hline $\mathrm{HC}(\mathrm{cm})$ & $100.1 \pm 10.0$ \\
\hline WHR & $0.76 \pm 0.06$ \\
\hline WSR & $0.47 \pm 0.07$ \\
\hline $\mathrm{BAI}(\%)$ & $30.6 \pm 5.4$ \\
\hline \multicolumn{2}{|c|}{$\begin{array}{l}\text { BMI, body mass index; BAI, body adiposity index; DBP, diastolic } \\
\text { blood pressure; HC, hip circumference; HDL cholesterol, } \\
\text { high-density lipoprotein cholesterol; SBP, systolic blood pressure } \\
\text { WC, waist circumference; WHR, waist-to-hip ratio; WSR, } \\
\text { waist-to-stature ratio. }\end{array}$} \\
\hline
\end{tabular}

SBP. The general CVD risk score model was also developed using data from the American Framingham Heart Study but using a larger cohort than the Framingham model. ${ }^{20}$ Individuals without CVD were used in the development of the general CVD risk score model. ${ }^{20}$ The simplified general CVD risk score model was developed similarly as the general CVD risk score model. It is, however, a simpler CVD risk prediction model which is calculated using non-laboratory predictors. Risk variables (age, SBP, current antihypertensive treatment, smoking status and diabetes status) were used in both models. ${ }^{20}$ The only difference is that, BMI is included in the simplified general CVD risk score model instead of total and HDL cholesterol which is used in the general CVD risk score model.

\section{Statistical analysis}

The data on the representative sample of 4487 Australian women were described using mean \pm SD for continuous variables, while counts and percentages were used for categorical variables. Non-parametric Spearman's rank correlation was used to assess the associations between anthropometric measurements of obesity with CVD risk factors, and with the calculated 10-year predicted risks, due to the skewness in the distribution of some variables. Anthropometric measurements were also converted to $\mathrm{z}$-scores (original value subtracted by the mean and result divided by the SD) to represent the number of SDs above and below the mean for each participant. Logistic regression was used to assess the effects of each standardised anthropometric measurement of being above the recommended treatment thresholds for various risk score models as a result of $1 \mathrm{SD}$ increment above the mean for each anthropometric measure of obesity. ORs and associated 95\% CIs represented the likelihood of being above the recommended treatment thresholds for the specific risk score models (20\% for the Framingham risk score model for 10-year CVD incidence or death; 10\% for SCORE risk chart for high-risk regions for 10-year CVD death; $10 \%$ and $20 \%$ for the general CVD and simplified general CVD risk score models for 10-year CVD incidence and death). The predictive ability of these anthropometric measures to identify individuals above and below the

Table 2 Frequency distribution of 10-year predicted CVD incidence and mortality using various risk prediction models, in incremental risk categories of $10 \%$

\begin{tabular}{|c|c|c|c|c|c|}
\hline & \multicolumn{5}{|c|}{ Risk categories } \\
\hline & $0-9 \%$ & $10-19 \%$ & $20-29 \%$ & $30-39 \%$ & $\geq 40 \%$ \\
\hline Framingham 10-year predicted risk for CVD incidence ${ }^{18}$ & $2936(67.0 \%)$ & $764(17.4 \%)$ & $417(9.5 \%)$ & $179(4.1 \%)$ & $89(2.0 \%)$ \\
\hline Framingham 10-year predicted risk for CVD death ${ }^{18}$ & $4354(99.3 \%)$ & $29(0.7 \%)$ & $2(0 \%)$ & $0(0 \%)$ & $0(0 \%)$ \\
\hline SCORE-HIGH 10-year predicted risk for CVD death ${ }^{19}$ & $4318(98.5 \%)$ & $53(1.2 \%)$ & $9(0.2 \%)$ & $4(0.1 \%)$ & $1(0 \%)$ \\
\hline $\begin{array}{l}\text { GCVD 10-year predicted risk for CVD incidence and } \\
\text { death }^{20}\end{array}$ & $3738(85.2 \%)$ & $503(11.5 \%)$ & $109(2.5 \%)$ & $21(0.5 \%)$ & $14(0.3 \%)$ \\
\hline $\begin{array}{l}\text { SGCVD 10-year predicted risk for CVD incidence and } \\
\text { death }^{20}\end{array}$ & $3809(85.7 \%)$ & $519(11.7 \%)$ & $90(2.0 \%)$ & $19(0.4 \%)$ & $9(0.2 \%)$ \\
\hline
\end{tabular}

Counts and percentages of women were presented.

CVD, cardiovascular disease; GCVD, general cardiovascular disease risk score model; SCORE-HIGH, SCORE risk chart for high-risk regions; SGCVD, simplified general cardiovascular disease risk score model. 
treatment thresholds was assessed using sensitivity, specificity and area under the receiver operating characteristic (ROC) curve. p Values of less than 0.05 were considered to be statistically significant. All statistical analyses were performed with IBM SPSS Statistics V.21.

\section{RESULTS}

The sample of 4487 women aged 20-69 years from the NHF Risk Factor Prevalence Study is a representative sample of the Australian female population, free of heart disease, diabetes and stroke. The characteristics of the sample are summarised in table 1 . In addition to the conventional risk factors for CVD, all anthropometric measurements of general and central obesity were presented.

The 10-year CVD risk of each participant in the sample was calculated using four risk score models. The frequency distribution of calculated risks is presented in table 2. Except for the Framingham model for CVD incidence, all other models predicted risks of less than $10 \%$ for at least $85 \%$ of the sample. The Framingham model for CVD incidence, general CVD model for CVD incidence and death and simplified general CVD model for CVD incidence and death predicted risk values across the entire range from $0 \%$ to greater than $40 \%$.

Anthropometric measurements of obesity were positively correlated with age, SBP, total cholesterol and total cholesterol to HDL cholesterol ratio (all Spearman's $r \geq 0.195$, $p<0.001$ ), with HC recording the lowest correlations. These obesity measures were negatively correlated with HDL cholesterol (all Spearman's $r \leq-0.160$, $\mathrm{p}<0.001)$. Measures of central obesity that included a measure of WC (WHR and WSR) generally recorded better correlations compared with measures of general obesity (BMI and BAI).

The associations between anthropometric measurements of obesity and the 10-year predicted risks calculated using the four models are presented in table 3. All Spearman's rank correlations were statistically significant $(p<0.0005)$. All anthropometric measures of central obesity (WC, WHR and WSR) generally had consistently higher correlations with the predicted risks calculated using the four CVD risk score models, as compared with measures of general obesity.

Recommended treatment thresholds for the four CVD risk models were identified from a review of the literature. Table 4 presents the effects of $1 \mathrm{SD}$ increment in each anthropometric measurement above the mean on the likelihood of being above the recommended thresholds or being indicated for treatment. All anthropometric measures of central obesity (WC, WHR and WSR) generally recorded higher ORs than general measures of obesity and they increased the likelihood of individuals being above the respective treatment thresholds.

Anthropometric measurements of central obesity (WC, WHR and WSR) also recorded higher area under the ROC curves, higher sensitivity and specificity, than BMI in identifying women above and below the $20 \%$ treatment threshold for the Framingham model for 10-year CVD incidence (figure 1A) and general CVD model for 10-year CVD incidence and death (figure 1B). Although BMI is included in the simplified general CVD model, high area under the ROC curve $(>0.76)$ are reported for WC and WHR (figure 1C), indicating the independent contribution of central obesity measurements as compared with general obesity measurement in predicting the increased risk of CVD.

\section{DISCUSSION}

Measures of obesity are generally not included in the prediction of CVD risk. BMI is the only measure of obesity currently included in CVD risk score models such as the simplified general CVD risk score model, as an alternative to total and HDL cholesterol level for ease of measurement and calculation, ${ }^{20}$ and in the QRISK score model. ${ }^{29}$

In our study, anthropometric measurements of central obesity (WC, WHR and WSR) were more strongly associated with conventional CVD risk factors and the 10-year predicted risk calculated using the Framingham risk score model, SCORE risk chart for high-risk regions, general CVD and simplified general CVD risk score

Table 3 Non-parametric correlations between anthropometric measurements of general and central obesity and 10-year predicted risk of CVD incidence and mortality in 4487 women

\begin{tabular}{|c|c|c|c|c|c|c|}
\hline & BMI & WC & $\mathrm{HC}$ & WHR & WSR & BAI \\
\hline Framingham 10-year predicted risk for CVD incidence ${ }^{18}$ & 0.380 & 0.450 & 0.301 & 0.409 & 0.485 & 0.378 \\
\hline Framingham 10-year predicted risk for CVD death ${ }^{18}$ & 0.394 & 0.452 & 0.307 & 0.404 & 0.483 & 0.377 \\
\hline SCORE-HIGH 10-year predicted risk for CVD death ${ }^{19}$ & 0.309 & 0.381 & 0.253 & 0.348 & 0.419 & 0.338 \\
\hline GCVD 10-year predicted risk for CVD incidence and death ${ }^{20}$ & 0.385 & 0.452 & 0.307 & 0.405 & 0.487 & 0.383 \\
\hline SGCVD 10-year predicted risk for CVD incidence and death ${ }^{20}$ & * & 0.446 & 0.320 & 0.384 & * & * \\
\hline
\end{tabular}

All Spearman's rank correlations significant at the $p<0.0005$ level.

${ }^{*}$ Correlation is not calculated for this obesity measure as it contains variables that are also used in the calculation of the simplified general CVD model.

BMI, body mass index; BAI, body adiposity index; CVD, cardiovascular disease; GCVD, general cardiovascular disease risk score model; $\mathrm{HC}$, hip circumference; WC, waist circumference; SCORE-HIGH, SCORE risk chart for high-risk regions; SGCVD, simplified general cardiovascular disease risk score model; WHR, waist-to-hip ratio; WSR, waist-to-stature ratio. 


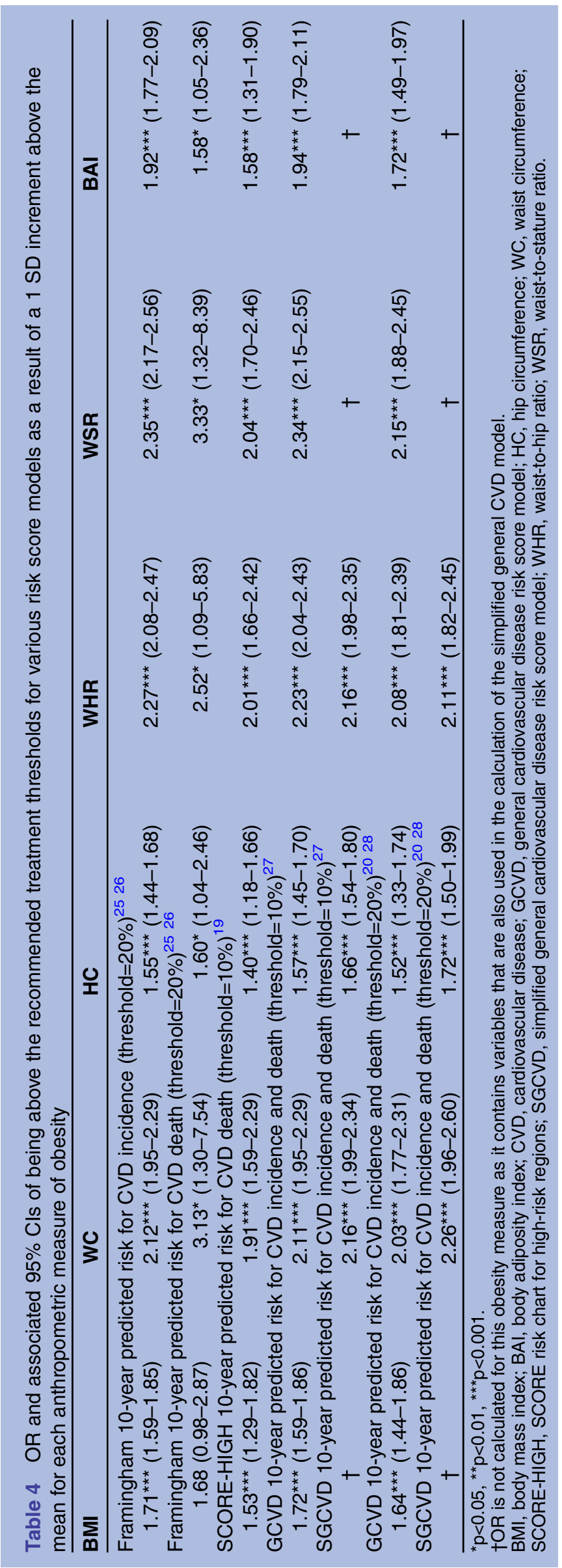

model, compared with general measures of obesity. Central obesity measures also recorded higher ORs and increased the likelihood of being above the recommended treatment threshold of the respective models with $1 \mathrm{SD}$ increase above the mean. Central obesity measures which incorporated the measure of WC also exhibited higher sensitivity and specificity than BMI. Although BMI is included in the calculation of the simplified general CVD model, high area under the ROC curves were reported for WC and WHR, thus confirming that anthropometric measures of central obesity independently and significantly predicts CVD risk that is not accounted for by the general obesity measure. Hence, $\mathrm{BMI}$ alone is insufficient to account for the association between obesity and CVD risk.

Consistent with our study findings, previous studies also reported stronger associations between central obesity measures and CVD risk. Higher standardised ORs adjusted for BMI were reported for WC and CVD, compared with BMI, in women from the International Day for the Evaluation of Abdominal Obesity (IDEA) study. ${ }^{30} 31$ An increase in WC was associated with being 4.25 times more likely of stroke and transient ischaemic attacks. ${ }^{32}$ Conversely, some studies reported that the association between BMI and CVD was similar to measures of central obesity. ${ }^{33} 34$

There are several possible explanations for our study findings that measures of central obesity are better predictors of CVD risk than BMI. Greater central obesity is associated with systemic inflammation which directly contributes to CVD risk. ${ }^{35}$ Hence, measures that account for the accumulation of excess abdominal fat would report stronger associations and are desirable for assessing adiposity. They would also be more accurate at indicating CVD risk and should be incorporated into CVD assessment. $^{36-39}$ The addition of central obesity measures to BMI has also been shown to improve the accuracy of stratifying participants into lower and higher risk categories for mortality ${ }^{40}$ and provides incremental value in predicting CVD above and beyond that provided by general obesity measures. ${ }^{41-45}$ BMI is a flawed measure as it does not correctly identify individuals with excess body fat due to its inability to differentiate fat and fat-free mass and it does not account for the effect of age and ethnicity on body fat distribution. ${ }^{46-50}$ An increase in muscle or fat-free mass would, however, be reflected in the central obesity measures.

Among central obesity measures, we found their performance to be comparable in our study. It remains unclear which measurement should be incorporated into CVD risk score models. A collaborative analysis of 58 prospective studies, however, reported that measures of general and central obesity did not improve CVD risk assessment when information is available on SBP, diabetes and lipids. ${ }^{51}$ Overweight and obesity are, nevertheless, important in CVD prevention, with one of three fatal and one of seven non-fatal CVD cases attributable to it. ${ }^{34}$

Opinion remains divided as to which is a more appropriate measurement for assessing adiposity and its association 
(a) Framingham model

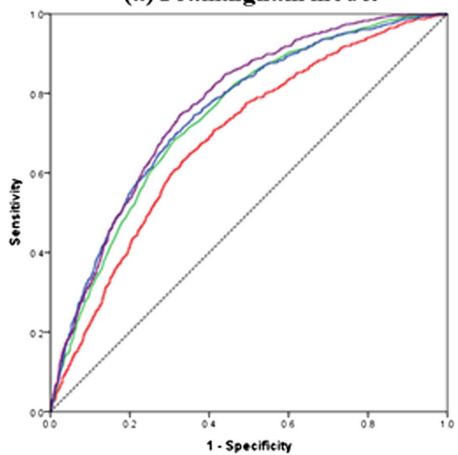

(b) General CVD model

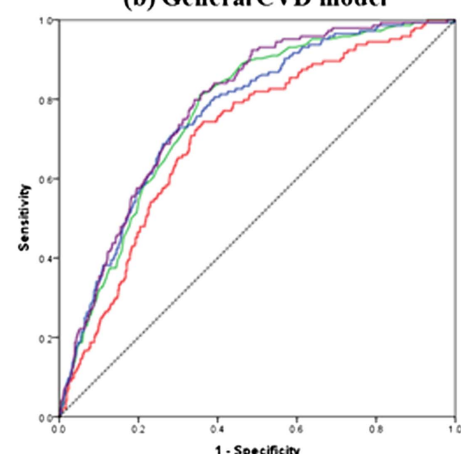

(c) Simplified general CVD model

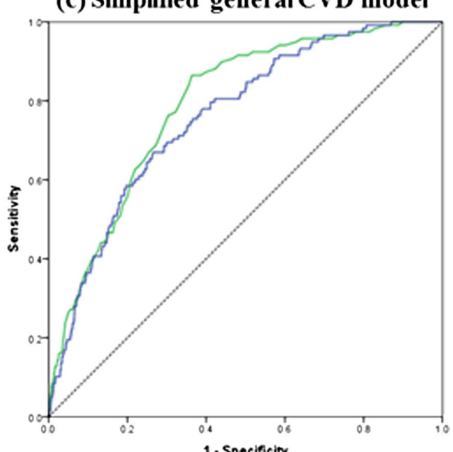

1. Specificity

\begin{tabular}{|c|c|c|c|c|}
\hline Obesity measures & Colour & (a) & (b) & (c) \\
\hline \multirow{2}{*}{ BMI } & Red & $\begin{array}{c}0.685 \\
(0.664,0.706)\end{array}$ & $\begin{array}{c}0.710 \\
(0.670,0.750)\end{array}$ & $\#$ \\
\hline \multirow{2}{*}{ WC } & Green & $\begin{array}{c}0.741 \\
(0.722,0.760)\end{array}$ & $\begin{array}{c}0.767 \\
(0.733,0.801)\end{array}$ & $\begin{array}{c}0.791 \\
(0.754,0.827)\end{array}$ \\
\hline \multirow{2}{*}{ WHR } & Blue & $\begin{array}{c}0.749 \\
(0.730,0.768)\end{array}$ & $\begin{array}{c}0.764 \\
(0.728,0.799)\end{array}$ & $\begin{array}{c}0.761 \\
(0.720,0.801)\end{array}$ \\
\hline \multirow{2}{*}{ WSR } & Purple & $\begin{array}{c}0.766 \\
(0.749,0.784)\end{array}$ & $(0.751,0.815)$ & $\#$ \\
\hline
\end{tabular}

Figure 1 ROC curves to compare the predictive ability of obesity measures for being above the $20 \%$ cut-off of three CVD models: (A) Framingham risk score model for 10-year CVD incidence; (B) general cardiovascular disease risk score model for 10-year CVD incidence and death; (C) simplified general cardiovascular disease risk score model for 10-year CVD incidence and death. \#Area under the ROC curve is not calculated for this obesity measure as it contains height which is also used in the calculation of the simplified general CVD model. BMI, body mass index; CVD, cardiovascular disease; ROC, receiver operating characteristic; WC, waist circumference; WHR, waist-to-hip ratio; WSR, waist-to-stature ratio.

with CVD risk. ${ }^{37}$ Some studies recommended the use of WC in clinical assessment and research studies. ${ }^{52}{ }^{53}$ In a systematic review and meta-analysis study of Caucasians without CVD, WC was most highly correlated with all CVD risk factors, compared with BMI, WHR, WSR and body fat percentage, in women. ${ }^{52}$ In other studies, WC was also more closely associated with CVD risk factors than other measures of central obesity and BMI in women. ${ }^{54-57}$ The advantages of $\mathrm{WC}$ are: it is easy to measure and interpret and it is less prone to measurement and calculation error. ${ }^{53}$ Appropriate sex, age and ethnic-specific WC cutpoints would need to be established. ${ }^{44}$ It would also be difficult to use WC in today's multicultural societies due to requirements for different cut-points. ${ }^{50}$

The use of WHR is also supported as it is less strongly associated with BMI than WC, and is thus a more specific surrogate for fat distribution. ${ }^{40}$ A longitudinal population study on 1462 women from Sweden reported stronger relations between WHR and CVD endpoints, compared with BMI, WC and HC. ${ }^{58}$ These relations were mostly independent of age, BMI and either SBP, cholesterol level or smoking habit. ${ }^{58}$ In a meta-regression analysis of prospective studies, WHR was also more strongly associated with CVD compared with WC, although the difference was not significant. ${ }^{37}$ Another study reported that WHR was associated with CVD mortality but not WC in elderly women from the UK. ${ }^{59}$ Elevated WHR was also independently associated with a higher CVD risk in the Nurses' Health Study and in the Swedish Women's Lifestyle and Health Cohort Study. ${ }^{45}{ }^{60}$ Women with a WHR of $\geq 0.88$ were 3.25 times more at risk of CHD compared with women with a WHR of $<0.72$ after adjusting for BMI and other CVD risk factors. ${ }^{45}$ Higher age-adjusted and sex-adjusted ORs were also reported with WHR and CHD and CVD mortality, compared with WC and BMI, in an Australian population without heart disease, diabetes or stroke. ${ }^{61}$ Similar results were presented in other studies. WHR reported the highest age standardised HRs in relation to CVD mortality, followed by WSR, WC and BMI in women. ${ }^{62} 63$ The advantages of WHR are: it has low measurement error, high precision and no bias over a wide range of ethnic groups. ${ }^{64}$ WHR, however, may not be suitable for assessing central obesity in the elderly ${ }^{65}$ due to laxity of abdominal muscles which would undermine the predictive value of abdominal circumferences. ${ }^{55}$ It is also more difficult to measure than WC. ${ }^{37}$ Despite its limitations, WHR has been recommended for incorporation into CVD risk assessment. ${ }^{37}$

WSR is the least commonly used measure of central obesity. In a systematic review and meta-analysis study, WSR reported the weakest correlations with CVD risk factors, compared with BMI and other measures of central obesity, ${ }^{52}$ which is contrary to our study findings. In contrast, WSR was most highly correlated with CHD risk predicted using the Framingham model ${ }^{18}$ in women from England, compared with BMI, WC and WHR in another study. ${ }^{66}$ WSR, however, reported lower correlations than WC and BMI following adjustments for age. ${ }^{66}$ The advantage of WSR is that the same cut-point could be applied across a wide range of populations. A cut-off value of 0.5 indicates increased risk for men and women and people of different ethnic groups, and this 
value may also be used in children and adults, unlike WC which requires different cut-offs. ${ }^{67}{ }^{68}$ More research is required to assess the association between WSR and CVD risk in women, in comparison with WC, WHR and BMI.

Our study has limitations. This study is cross sectional; however, it is a representative sample of the Australian female population. There is only one set of baseline measurements recorded for some risk variables but important variables including anthropometric measures of obesity are measured twice. Further, the 10-year CVD risks are calculated using risk score models to stratify individuals against the treatment thresholds of the various models, and are not prospective CVD events.

\section{CONCLUSIONS}

Central obesity is more strongly associated with CVD risk than general obesity. The deposition of adipose tissue is associated with systemic inflammation which has a direct effect on CVD risk. Therefore, increments in central obesity have a more detrimental effect on CVD risk compared with increments in general obesity.

When used alone, BMI is inadequate for identifying individuals at increased risk of CVD as it does not differentiate between fat and fat-free mass. On the other hand, anthropometric measurements of central obesity have higher sensitivity and specificity. These measures are also more sensitive to lifestyle modifications. An increase in muscle mass through diet and training would lead to changes in measures such as WC and WSR but little change might be indicated with BMI. ${ }^{69}$ It would be more useful to measure a patient's central obesity during clinical assessment to evaluate the effect of lifestyle changes in relation to CVD risk compared with BMI. Central obesity measures are also significant and independent predictors of CVD risk, accounting for additional risk above BMI. These measurements should be incorporated into CVD risk assessment, particularly when assessing the risk in women and the elderly. ${ }^{53} 70-73$

Future prospective studies are required to elucidate which anthropometric measurements of central obesity are better indicators or predictors of CVD risk. ${ }^{69}$ Studies measuring body fat distribution using CT or MRI are desirable to better understand the association between body fat distribution and mortality, but are costly. ${ }^{74}$

In conclusion, WC, WHR and WSR, or measures of central obesity that include a measurement of $\mathrm{WC}$, should be considered for incorporation into the clinical assessment of CVD risk. Treatment of well-established CVD risk factors coupled with reducing overweight and obesity through lifestyle modifications would be an advisable goal in the primary prevention of CVD. ${ }^{4}$ It is equally important to maintain a healthy weight and to prevent central or abdominal obesity concurrently.

Acknowledgements Curtin University provided educational support to LGHG through the Curtin International Postgraduate Research Scholarship.

Contributors LGHG was involved in drafting the manuscript, interpretation of the data and revising the manuscript critically for important intellectual content. SSD conceived the study, performed the analysis and data interpretation and revised the manuscript critically for important intellectual content. TAW participated in the study design, acquired the data and revised the manuscript critically for important intellectual content. All authors read and approved the final manuscript.

Funding This research received no specific grant from any funding agency in the public, commercial or not-for-profit sectors.

\section{Competing interests None.}

\section{Patient consent Obtained.}

Ethics approval Australian Institute of Health Interim Ethics Committee and Human Research Ethics Committee at Curtin University.

Provenance and peer review Not commissioned; externally peer reviewed.

Data sharing statement No additional data are available.

Open Access This is an Open Access article distributed in accordance with the Creative Commons Attribution Non Commercial (CC BY-NC 3.0) license, which permits others to distribute, remix, adapt, build upon this work noncommercially, and license their derivative works on different terms, provided the original work is properly cited and the use is non-commercial. See: http:// creativecommons.org/licenses/by-nc/3.0/

\section{REFERENCES}

1. World Health Organization. Obesity and overweight. Secondary obesity and overweight, 2012. http://www.who.int/mediacentre/ factsheets/fs311/en/

2. Third Report of the National Cholesterol Education Program (NCEP). Expert panel on detection, evaluation, and treatment of high blood cholesterol in adults (Adult Treatment Panel III) final report. Circulation 2002;106:3143-421.

3. Kannel WB. Metabolic risk factors for coronary heart disease in women: perspective from the Framingham study. Am Heart $J$ 1987;114:413-19

4. Hubert HB, Feinleib M, McNamara PM, et al. Obesity as an independent risk factor for cardiovascular disease: a 26-year follow-up of participants in the Framingham Heart Study. Circulation 1983;67:968-77.

5. Manson JE, Colditz GA, Stampfer MJ, et al. A prospective study of obesity and risk of coronary heart disease in women. $N$ Engl J Med 1990;322:882-9.

6. Manson JE, Willett WC, Stampfer MJ, et al. Body weight and mortality among women. N Engl J Med 1995;333:677-85.

7. Dorn JM, Schisterman EF, Winkelstein W, et al. Body mass index and mortality in a general population sample women of men and women: the Buffalo Health Study. Am J Epidemiol 1997;146:919-31.

8. Cornier M-A, Després J-P, Davis N, et al. Assessing adiposity: a scientific statement from the American Heart Association. Circulation 2011;124:1996-2019.

9. Jensen MD, Ryan DH, Apovian CM, et al. 2013 AHA/ACC/TOS guideline for the management of overweight and obesity in adults: a report of the American College of Cardiology/American Heart Association Task Force on Practice Guidelines and The Obesity Society. Circulation 2013.

10. Park YS, Kim J-S. Obesity phenotype and coronary heart disease risk as estimated by the Framingham risk score. J Korean Med Sci 2012;27:243-9.

11. Satoh $\mathrm{H}$, Kishi $\mathrm{R}$, Tsutsui $\mathrm{H}$. Body mass index can similarly predict the presence of multiple cardiovascular risk factors in middle-aged Japanese subjects as waist circumference. Intern Med 2010;49:977-82.

12. Ryan MC, Fenster Farin HM, Abbasi F, et al. Comparison of waist circumference versus body mass index in diagnosing metabolic syndrome and identifying apparently healthy subjects at increased risk of cardiovascular disease. Am J Cardiol 2008;102:40-6.

13. Ying $\mathrm{X}$, Song $\mathrm{Z}$, Zhao $\mathrm{C}$, et al. Body mass index, waist circumference, and cardiometabolic risk factors in young and middle-aged Chinese women. J Zhejiang Univ Sci B 2010;11:639-46.

14. Zhu S, Heymsfield SB, Toyoshima $\mathrm{H}$, et al. Race-ethnicity-specific waist circumference cutoffs for identifying cardiovascular disease risk factors. Am J Clin Nutr 2005;81:409-15.

15. Huang K-C, Lee M-S, Lee S-D, et al. Obesity in the elderly and its relationship with cardiovascular risk factors in Taiwan. Obes Res 2005;13:170-8. 
16. Bergman RN, Stefanovski D, Buchanan TA, et al. A better index of body adiposity. Obesity (Silver Spring) 2011;19:1083-9.

17. Dhaliwal SS, Welborn TA. Central obesity and multivariable cardiovascular risk as assessed by the Framingham prediction scores. Am J Cardiol 2009;103:1403-7.

18. Anderson KM, Odell PM, Wilson PW, et al. Cardiovascular disease risk profiles. Am Heart J 1991;121(1 Part 2):293-8.

19. Conroy RM, Pyörälä K, Fitzgerald AP, et al. Estimation of ten-year risk of fatal cardiovascular disease in Europe: the SCORE project. Eur Heart J 2003;24:987-1003.

20. D'Agostino RB, Vasan RS, Pencina MJ, et al. General cardiovascular risk profile for use in primary care-the Framingham Heart study. Circulation 2008;117:743-53.

21. Australian Risk Factor Prevalence Study Management Committee. Survey No. 3 1989. Canberra: National Heart Foundation of Australia and Australia Institute of Health, 1990.

22. Boyle CA, Dobson AJ, Egger G, et al. Waist-to-hip ratios in Australia: a different picture of obesity. Aust J Nutr Diet 1993;50:57-64.

23. Alexander H, Dugdale A. Which waist-hip ratio? Med J Aust 1990;153:367-8.

24. Cooney MT, Dudina A, De Bacquer D, et al. How much does HDL cholesterol add to risk estimation? A report from the SCORE investigators. Eur J Cardiovasc Prev Rehabil 2009;16:304-14.

25. Neil HAW, Perera R, Armitage JM, et al. Estimated 10-year cardiovascular risk in a British population: results of a national screening project. Int J Clin Pract 2008;62:1322-31.

26. Woodward M, Brindle $\mathrm{P}$, Tunstall-Pedoe $\mathrm{H}$. Adding social deprivation and family history to cardiovascular risk assessment: the ASSIGN score from the Scottish Heart Health Extended Cohort (SHHEC) Heart 2007;93:172-6.

27. Mosca L, Benjamin EJ, Berra K, et al. Effectiveness-based guidelines for the prevention of cardiovascular disease in women2011 update a guideline from the American Heart Association. J Am Coll Cardiol 2011;57:1404-23.

28. Genest J, McPherson R, Frohlich J, et al. 2009 Canadian Cardiovascular Society/Canadian guidelines for the diagnosis and treatment of dyslipidemia and prevention of cardiovascular disease in the adult-2009 recommendations. Can J Cardiol 2009;25:567-79.

29. Goh LGH, Dhaliwal SS, Lee AH, et al. Utility of established cardiovascular disease risk score models for the 10-year prediction of disease outcomes in women. Expert Rev Cardiovasc Ther 2013;11:425-35.

30. Wittchen H-U, Balkau B, Massien C, et al. International day for the evaluation of abdominal obesity: rationale and design of a primary care study on the prevalence of abdominal obesity and associated factors in 63 countries. Eur Heart J Suppl 2006;8(Suppl B):B26-33.

31. Balkau B, Deanfield JE, Despres JP, et al. International day for the evaluation of abdominal obesity (IDEA) - a study of waist circumference, cardiovascular disease, and diabetes mellitus in 168 000 primary care patients in 63 countries. Circulation 2007;116:1942-51.

32. Winter Y, Rohrmann S, Linseisen J, et al. Contribution of obesity and abdominal fat mass to risk of stroke and transient ischemic attacks. Stroke 2008;39:3145-51.

33. Taylor AE, Ebrahim S, Ben-Shlomo Y, et al. Comparison of the associations of body mass index and measures of central adiposity and fat mass with coronary heart disease, diabetes, and all-cause mortality: a study using data from 4 UK cohorts. Am J Clin Nutr 2010;91:547-56.

34. van Dis I, Kromhout D, Geleijnse JM, et al. Body mass index and waist circumference predict both 10-year nonfatal and fatal cardiovascular disease risk: study conducted in 20000 Dutch men and women aged 20-65 years. Eur J Cardiovasc Prev Rehabil 2009;16:729-34.

35. Berg AH, Scherer PE. Adipose tissue, inflammation, and cardiovascular disease. Circ Res 2005;96:939-49.

36. Snijder MB, van Dam RM, Visser M, et al. What aspects of body fat are particularly hazardous and how do we measure them? Int $J$ Epidemiol 2006;35:83-92.

37. de Koning L, Merchant AT, Pogue J, et al. Waist circumference and waist-to-hip ratio as predictors of cardiovascular events: meta-regression analysis of prospective studies. Eur Heart $J$ 2007;28:850-6.

38. Dalton M, Cameron AJ, Zimmet PZ, et al. Waist circumference waist-hip ratio and body mass index and their correlation with cardiovascular disease risk factors in Australian adults. J Intern Med 2003;254:555-63

39. Antillon D, Towfighi A. No time to 'weight': the link between obesity and stroke in women. Womens Health 2011;7:453-63.
40. Pischon $\mathrm{T}$, Boeing $\mathrm{H}$, Hoffmann $\mathrm{K}$, et al. General and abdominal adiposity and risk of death in Europe. $N$ Engl J Med 2008;359:2105-20.

41. Li C, Engstrom G, Hedblad B, et al. Sex differences in the relationships between BMI, WHR and incidence of cardiovascular disease: a population-based cohort study. Int $J$ Obes 2006;30:1775-81.

42. Freiberg MS, Pencina MJ, D'Agostino RB, et al. BMI vs. waist circumference for identifying vascular risk. Obesity 2008;16:463-9.

43. DiPietro L, Katz LD, Nadel ER. Excess abdominal adiposity remains correlated with altered lipid concentrations in healthy older women. Int J Obes Relat Metab Disord 1999;23:432-6.

44. Klein S, Allison DB, Heymsfield SB, et al. Waist circumference and cardiometabolic risk: a consensus statement from shaping America's health: association for weight management and obesity prevention NAASO, The Obesity Society; the American Society for Nutrition; and the American Diabetes Association. Obesity 2007;15:1061-7.

45. Rexrode KM, Carey VJ, Hennekens $\mathrm{CH}$, et al. Abdominal adiposity and coronary heart disease in women. JAMA 1998;280:1843-8.

46. Fogelholm M. Physical activity, fitness and fatness: relations to mortality, morbidity and disease risk factors. A systematic review. Obes Rev 2010;11:202-21.

47. Romero-Corral A, Somers VK, Sierra-Johnson J, et al. Accuracy of body mass index in diagnosing obesity in the adult general population. Int J Obes 2008;32:959-66.

48. World Health Organization. Obesity: preventing and managing the global epidemic. Report of a WHO consultation. World Health Organ Tech Rep Ser: WHO, 2000

49. Deurenberg P, Yap M, van Staveren WA. Body mass index and percent body fat: a meta-analysis among different ethnic groups. Int $J$ Obes 1998;22:1164-71.

50. Welborn TA, Dhaliwal SS. Being correct about obesity. Med J Aust 2011;194:429-30

51. The Emerging Risk Factors Collaboration. Separate and combined associations of body-mass index and abdominal adiposity with cardiovascular disease: collaborative analysis of 58 prospective studies. Lancet 2011;377:1085-95.

52. Dijk SB, Takken T, Prinsen EC, et al. Different anthropometric adiposity measures and their association with cardiovascular disease risk factors: a meta-analysis. Neth Heart J 2012;20:208-18.

53. Dobbelsteyn CJ, Joffres MR, MacLean DR, et al. A comparative evaluation of waist circumference, waist-to-hip ratio and body mass index as indicators of cardiovascular risk factors. The Canadian Heart Health Surveys. Int J Obes 2001;25:652-61.

54. Pouliot M-C, Després J-P, Lemieux S, et al. Waist circumference and abdominal sagittal diameter: best simple anthropometric indexes of abdominal visceral adipose tissue accumulation and related cardiovascular risk in men and women. Am J Cardiol 1994;73:460-8.

55. Turcato E, Bosello O, Di Francesco V, et al. Waist circumference and abdominal sagittal diameter as surrogates of body fat distribution in the elderly: their relation with cardiovascular risk factors. Int J Obes Relat Metab Disord 2000;24:1005-10.

56. Zhu S, Wang Z, Heshka S, et al. Waist circumference and obesity-associated risk factors among whites in the third National Health and Nutrition Examination Survey: clinical action thresholds. Am J Clin Nutr 2002;76:743.

57. Reeder BA, Senthilselvan A, Després JP, et al. The association of cardiovascular disease risk factors with abdominal obesity in Canada. Canadian Heart Health Surveys Research Group. CMAJ 1997;157(Suppl 1):S39-45.

58. Lapidus L, Bengtsson C, Larsson B, et al. Distribution of adipose tissue and risk of cardiovascular disease and death: a 12 year follow up of participants in the population study of women in Gothenburg, Sweden. BMJ 1984;289:1257-61.

59. Price GM, Uauy $\mathrm{R}$, Breeze $\mathrm{E}$, et al. Weight, shape, and mortality risk in older persons: elevated waist-hip ratio, not high body mass index, is associated with a greater risk of death. Am J Clin Nutr 2006;84:449-60.

60. Lu M, Ye W, Adami HO, et al. Prospective study of body size and risk for stroke amongst women below age 60. J Intern Med 2006;260:442-50.

61. Dhaliwal SS, Welborn TA. Central obesity and cigarette smoking are key determinants of cardiovascular deaths in Australia: a public health perspective. Prev Med 2009;49:153-7.

62. Welborn TA, Dhaliwal SS. Preferred clinical measures of central obesity for predicting mortality. Eur J Clin Nutr 2007;61:1373-9.

63. Welborn TA, Dhaliwal SS, Bennett SA. Waist-hip ratio is the dominant risk factor predicting cardiovascular death in Australia. Med J Aust 2003;179:580-5. 
64. Dhaliwal SS, Welborn TA. Measurement error and ethnic comparisons of measures of abdominal obesity. Prev Med 2009;49:148-52.

65. Goodman-Gruen D, Barrett-Connor E. Sex differences in measures of body fat and body fat distribution in the elderly. Am J Epidemiol 1996;143:898-906.

66. Ashwell M, Lejeune S. Ratio of waist circumference to height may be better indicator of need for weight management. BMJ 1996; 312:377.

67. Ashwell M, Hsieh SD. Six reasons why the waist-to-height ratio is a rapid and effective global indicator for health risks of obesity and how its use could simplify the international public health message on obesity. Int J Food Sci Nutr 2005;56:303-7.

68. Browning LM, Hsieh SD, Ashwell M. A systematic review of waist-to-height ratio as a screening tool for the prediction of cardiovascular disease and diabetes: $0 \mathbf{} 5$ could be a suitable global boundary value. Nutr Res Rev 2010;23:247-69.
69. Schneider HJ, Glaesmer H, Klotsche J, et al. Accuracy of anthropometric indicators of obesity to predict cardiovascular risk. $J$ Clin Endocrinol Metab 2007;92:589-94.

70. Okosun IS, Liao Y, Rotimi CN, et al. Abdominal adiposity and clustering of multiple metabolic syndrome in White, Black and Hispanic Americans. Ann Epidemiol 2000;10:263-70.

71. Ho SC, Chen YM, Woo JLF, et al. Association between simple anthropometric indices and cardiovascular risk factors. Int $J$ Obes Relat Metab Disord 2001;25:1689-97.

72. Jeong S-K, Seo M-W, Kim Y-H, et al. Does waist indicate dyslipidemia better than BMI in Korean adult population? J Korean Med Sci 2005;20:7-12.

73. Yusuf S, Hawken S, Ôunpuu S, et al. Obesity and the risk of myocardial infarction in 27000 participants from 52 countries: a case-control study. Lancet 2005;366:1640-9.

74. Moore SC. Waist versus weight-which matters more for mortality? Am J Clin Nutr 2009;89:1003-4. 